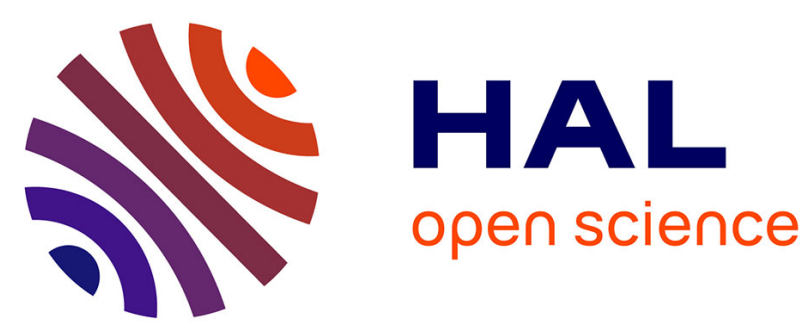

\title{
PARAMETRIC STUDIES OF WAVE PROPAGATION THROUGH IMPERFECT INTERFACES USING MICROMECHANICS BASED EFFECTIVE STIFFNESS
}

\author{
Anil Misra, Orestes Marangos
}

\section{To cite this version:}

Anil Misra, Orestes Marangos. PARAMETRIC STUDIES OF WAVE PROPAGATION THROUGH IMPERFECT INTERFACES USING MICROMECHANICS BASED EFFECTIVE STIFFNESS. 2011. hal-00556109

\author{
HAL Id: hal-00556109 \\ https://hal.science/hal-00556109
}

Preprint submitted on 18 Jan 2011

HAL is a multi-disciplinary open access archive for the deposit and dissemination of scientific research documents, whether they are published or not. The documents may come from teaching and research institutions in France or abroad, or from public or private research centers.
L'archive ouverte pluridisciplinaire HAL, est destinée au dépôt et à la diffusion de documents scientifiques de niveau recherche, publiés ou non, émanant des établissements d'enseignement et de recherche français ou étrangers, des laboratoires publics ou privés. 


\title{
PARAMETRIC STUDIES OF WAVE PROPAGATION THROUGH IMPERFECT INTERFACES USING MICROMECHANICS BASED EFFECTIVE STIFFNESS
}

\author{
Anil Misra $^{1}$ and Orestes Marangos ${ }^{1}$ \\ ${ }^{1}$ Department of Civil and Mechanical Engineering, University of Missouri-Kansas \\ City, Kansas City, MO 64110
}

\begin{abstract}
Plane wave propagation through contact between solids has been investigated using imperfectly bonded interface model. In this model, the contact behavior is represented through effective interface stiffnesses. We have developed a micromechanical methodology for determining complex-valued effective normal and shear interface stiffnesses by considering asperity contact interactions. The methodology incorporates effects of surface roughness, anisotropy, existing stress conditions and rate-dependent asperity contacts. We use the derived effective stiffnesses to elucidate wave transmission phenomena exhibited by interfaces.
\end{abstract}

Keywords: Acoustics, Interfaces, Reflection, Transmission, Effective Stiffness

PACS: $43.20 . \mathrm{E} 1,43.25$.Ts

\section{INTRODUCTION}

Plane wave transmission through contact between rough solids is of significance in the development of non-destructive evaluation methods for fractures, welds, and adhesive bonds. Imperfectly bonded interface models have been widely used to investigate the plane wave propagation through contact between two rough solids [see for example 1-3]. The assumption which forms the basis of the wave propagation models based upon the concept of imperfectly bonded interfaces is that the wavelength is much larger than the asperity contact size and asperity contact separation. Therefore, in these models, the contact behavior is represented through effective interface stiffnesses corresponding to interface length scales smaller than the size of wavelength should be considered. In these cases, the effective interface stiffness may be obtained by averaging asperity contact stiffnesses at sub-wavelength scales. Micromechanical approaches that explicitly include interface surface topography and incorporate material mechanical properties and intrinsic friction may be utilized to obtain the overall interface stiffness.

The authors have developed a micromechanical methodology for modeling contact behavior that incorporates the effects of surface roughness, anisotropy, existing stress conditions and rate-dependent asperity contact force laws. This methodology is applied to determine complex-valued effective normal and shear stiffnesses of interfaces which are explicit functions of surface geometry and stress conditions. The surface roughness for example can result in an inherently anisotropic interface. Similarly, rate-dependent 
asperity force laws result in effective stiffnesses that have complicated frequencydependency. These findings have critical implication on the interpretation of wave propagation through rough interfaces. We find that the amplitudes of the reflected and transmitted waves are significantly influenced by the interface roughness, and anisotropy. We also find that the frequency dependence of reflected and transmitted wave amplitudes is significantly different for rate-dependent and rate-independent interfaces. The micromechanical model may thus be used to elucidate the results of wave transmission through rough interfaces.

In the subsequent discussion, we first briefly describe the imperfectly bonded interface model. We then describe the essence of the micromechanical methodology and employ this model to study the behavior of wave transmission and reflection.

\section{IMPERFECTLY BONDED INTERFACE MODEL}

The imperfectly bonded interface model, also known as linear slip or displacement discontinuity approach [1-3], has been widely used to investigate wave propagation through interfaces between solids. In order to define the appropriate quantities and for completeness of our discussion, we give a brief description of the imperfectly bonded interface model. For convenience, we choose a coordinate system, such that the direction of incident wave propagation is within the 1-3 plane shown in Fig. 1.

At an imperfect interface between two media, tractions at upper medium $\mathrm{A}$ and lower medium B are continuous and displacements are discontinuous, which lead to the following equations where $n=0-3$ and $n=4-6$ are the wave modes in the incident and transmitting media, respectively.

$$
\begin{gathered}
\sum_{n=0}^{3} \sigma_{i 1}^{(n) A}=\sum_{n=4}^{6} \sigma_{i 1}^{(n) B} \\
\sum_{n=4}^{6} \sigma_{i 1}^{(n) B}=\left(\sum_{n=4}^{6} u_{j}^{(n)}-\sum_{n=0}^{3} u_{j}^{(n)}\right) C_{i j}
\end{gathered}
$$

We note that the interface overall traction, $F_{i}$, and the relative motion of the interface, $\delta_{j}$, are given as

$$
F_{i}=\sum_{n=4}^{6} \sigma_{i 1}^{(n) B}, \delta_{j}=\sum_{n=4}^{6} u_{j}^{(n)}-\sum_{n=0}^{3} u_{j}^{(n)}
$$

Equations (1), (2), and (3) may be combined to obtain the amplitudes of the reflected and transmitted $\mathrm{P}$ and $\mathrm{S}$ waves for the interface as follows

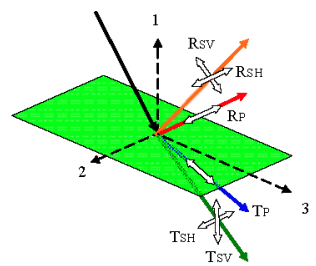

FIGURE 1. A schematic of wave reflection and transmission at an imperfect interface. 


$$
\left[\begin{array}{l:l}
{[A]} & {[B]} \\
\hdashline[D] & {[E]}
\end{array}\right]\left\{\begin{array}{l}
\{R\} \\
\hdashline\{T\}
\end{array}\right\}=\left\{\begin{array}{l}
\{C\} \\
\{\bar{F}\}
\end{array}\right\}
$$

where the sub-matrices [A], [B], [D] and [E] are a function of elastic constants of upper and lower media, and the nonlinear, stress-dependent interface stiffnesses derived from the micromechanical model described in the next section. Thus, the solution of (4) generates complex valued reflection, $\left\{R_{\}}=<R_{P}, R_{S V}, R_{S H}>\right.$, and transmission, $\{T\}=<T_{P}, T_{S V}, T_{S H}>$, coefficients which are related to interface stresses and roughness.

\section{MICROMECHANICS BASED EFFECTIVE STIFFNESS FOR INTERFACES}

A micromechanical methodology for the determination of the relationship between interface stress and deformation has been presented previously in Misra [4-5] by considering the force-deformation behavior of the asperities. In the micromechanical methodology, the stress-deformation behavior of an interface is obtained by considering the force-deformation behavior of the asperity contacts and the statistical description of the interface topography. At the asperity contact-level, a local force-deformation relationship is defined that accounts for the elastic deformation and inelastic sliding at the contact. As schematically depicted in Fig. 2, the stress-deformation relationship for an interface is then derived by utilizing: (1) the distribution functions of asperity heights and contact orientations, and (2) the overall kinematic constraints and equilibrium conditions.

The micromechanical methodology developed by the author extends other similar models [see 6 for a review] in the following ways by: (1) introducing a directional distribution function of asperity contact orientations as an additional measure of surface roughness recognizing that the asperity contacts are not equally likely in all directions, (2) using rate-dependent asperity force laws, and (3) using kinematic and/or static constraints to relate the asperity force/displacement to the overall interface stress/displacement, (4) using an iterative procedure to obtain the asperity contact forces/displacement at each load increment, recognizing that the asperity contact force distribution is not known a priori, and (5) introducing evolution laws for asperity heights and asperity contact orientations that account for the change in surface roughness resulting from asperity damage. In this paper, we give a derivation for linear rate-dependent interface behavior that can be utilized in the imperfect interface model to study wave propagation.

\section{$\underline{\text { Rate-Dependent Asperity Contact Behavior }}$}

The asperity contact forces, $f_{i}^{c}$, are related via the asperity contact stiffnesses, $K_{i j}{ }^{c}$, and asperity contact viscosities, $\eta_{i j}$, to asperity contact displacements and displacement rates as follows

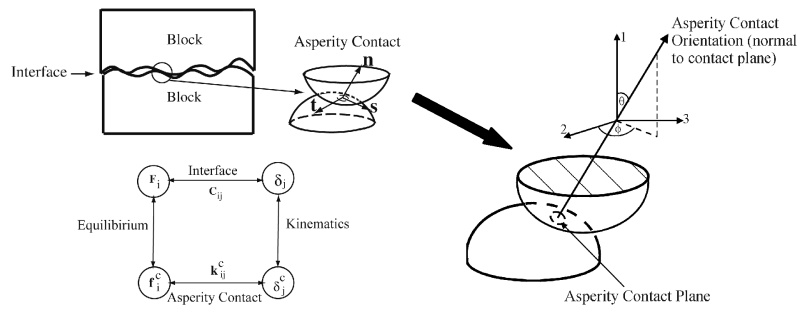

FIGURE 2. A schematic of the micromechanical methodology. 


$$
f_{i}^{c}=K_{i j}^{c} \delta_{j}^{c}+\eta_{i j}^{c} \delta_{j}^{k}
$$

The asperity contact stiffnesses and viscosities generally depend upon the contact loading condition, such as the stiffness given by the Hertzian contact theory. However, for the purposes of this paper, we utilize loading independent stiffnesses and viscosities. It is convenient to express the asperity stiffness tensor, $K_{i j}^{c}$, in terms of asperity stiffness that describes the behavior along the direction of normal and tangent to an asperity contact, such that

$$
K_{i j}^{c}=K_{n}^{c} n_{i}^{c} n_{j}^{c}+K_{s}^{c}\left(s_{i}^{c} s_{j}^{c}+t_{i}^{c} t_{j}^{c}\right)
$$

where $K_{n}$ and $K_{s}$ denote nonlinear asperity stiffness along the normal and tangential direction of the asperity. The unit vector $\mathbf{n}$ is normal to the asperity contact surface and vectors $\mathbf{s}$ and $\mathbf{t}$ are arbitrarily chosen on the plane tangential to the asperity contact surface, such that nst forms a local Cartesian coordinate system. It is noted that the stiffness term that cross-link normal and shear behavior are assumed to be negligible in accordance with the theories for contact of smooth non-conforming bodies. Similarly, the asperity viscosity tensor, $\eta_{i j}$, may be written as:

$$
\eta_{i j}^{c}=\eta_{n}^{c} n_{i}^{c} n_{j}^{c}+\eta_{s}^{c}\left(s_{i}^{c} s_{j}^{c}+t_{i}^{c} t_{j}^{c}\right)
$$

\section{Statistical Description of Interface Geometry}

The interface geometry determines the orientations and the number of asperity contacts under a given loading condition. The composite topography of contacting surfaces, described via statistics of asperity contact heights, orientations, and curvatures, may be utilized for this purpose [7-8]. In this paper, the statistical distribution of asperity contact heights is described via gamma distributions, and that of asperity contact orientation via spherical harmonic expansions. It is usual to define the asperity contact height with reference to the highest peak of the composite topography such that, asperity height, $r$, represents the overlap of the interacting surfaces. Surfaces that have smaller average asperity height and narrow distributions of asperity heights are considered to be relatively smoother. A density function for asperity heights denoted by, $H(r)$, can be used to model the height distributions. Thus, for an interface with $N$ asperities per unit area, $N$ $H(r) d r$, denotes that number of asperity contacts in the interval represented by $r$ and $r+d r$. Thus, the total number of asperity contacts, under a given loading condition, is given by

$$
N_{r}=\int_{0}^{r} N H(r) d r
$$

where $r$ represents the interface closure under a given loading. The asperity contact orientation is defined by considering the inclination of the asperity contact normal with respect to that of the interface normal direction. As shown in Fig. 2, the orientation of an oblique asperity contact is defined by the azimuthal angle $\phi$ and the meridional angle $\theta$, measured with respect to a Cartesian coordinate system in which direction 1 is normal to the interface. A 3-dimensional density function utilizing spherical harmonics expansion in spherical polar coordinates that describes the concentrations of asperity contact 
orientations was introduced by [4-5]. For an interface with isotropic geometry, the density function, $\xi(\Omega)$, of asperity contact orientations distribution in the domain: $\theta \leq \theta \leq \pi / 2 a, 0 \leq$ $\phi \leq 2 \pi$, is given by

$$
\xi(\Omega)=\frac{a \sin a \theta}{2 \pi \sin \theta} ? ? ?+\frac{b}{4}(3 \cos 2 a \theta+1)+3 c \sin ^{2} a \theta \cos 2 \phi_{?}^{?} \quad\left(0 \leq \theta \leq \frac{\pi}{2 a} ; \quad 0 \leq \phi \leq 2 \pi ; \quad a \geq 1\right)
$$

where angles $\phi$ and $\theta$ are defined in Fig. 2, $\Omega$ represents the solid angle formed by $\phi$ and $\theta$, and parameter $a$ determines the shape of the density function $\xi(\Omega)$. Thus, the product $N_{r} \xi(\Omega) d \Omega$ denotes the number of asperity contacts $N_{\Omega}$ in the interval represented by solid angles $\Omega$ and $\Omega+d \Omega$, that is

$$
N_{\Omega}=N_{r} \xi(\Omega) d \Omega
$$

The density function in (10) has the ability to model surfaces with varying roughness. As discussed in [5], the asperity contacts for smooth interfaces have a greater tendency to concentrate in the direction normal to the interface than that for rough interfaces. It is noteworthy that, as parameter, $a$, increases, the contact distribution concentrates towards the direction normal to the interface. In particular, the density function, $\xi(\Omega)$, behaves like a delta function in the limit $a \rightarrow \infty$ and yields an expectation $E[\theta]=0$, which represents a concentrated contact orientation, normal to the interface of a perfectly smooth joint. In general, the parameter, $a$, describes the extent of the asperity contacts in the meridional direction as well as the mean asperity contact orientation. Parameter, c, on the other hand, describes asperity concentration in the azimuthal directions.

\section{Overall Interface Stiffness}

The overall stiffness of the interface can be derived through two approaches, referred here as (1) the kinematic approach, and (2) the static approach. In the kinematic approach, we consider the equilibrium of forces. Consequently, the overall traction $F_{i}$ on the interface is obtained from the summation of the forces, $f_{i}^{c}$, developed at asperities, which for a large number of asperity contacts is written as the following integral:

$$
F_{i}=N \iint_{\Omega} f_{i}^{c} \xi(\Omega) H(r) d \Omega d r
$$

where the traction $F_{i}$ is given as force per unit area since $\mathrm{N}$ is measured per unit area of an interface. Under the kinematic assumption the relative motion at an asperity is same as the relative motion of the interface, that is

$$
\delta_{j}^{c}=\Delta_{j} \text { and } \dot{\delta}_{j}^{c}=\dot{\Delta}_{j}
$$

the overall traction, $F_{i}$ may be written as:

$$
F_{i}=C_{i j} \Delta_{j}+\eta_{i j} \dot{\Delta}_{j}
$$

where interface stiffness and viscosity tensors, denoted by, $C_{i j}$ and $\eta_{i j}$, respectively, are obtained as the following integrals: 
$C_{i j}=N \int_{0}^{r} \int_{0}^{2 \pi} \int_{0}^{\pi / 2 a} K_{i j}^{c} \xi(\phi, \theta) \sin \theta d \theta d \phi H(r) d r$ and $\eta_{i j}=N \int_{0}^{r} \int_{0}^{2 \pi} \int_{0}^{\pi / 2 a} \eta_{i j}^{c} \xi(\phi, \theta) \sin \theta d \theta d \phi H(r) d r$

where $\xi(\phi, \theta)$ is the asperity contact orientation distribution given by equation (9), and H(r) is asperity height distribution given by equation (8), $r=r_{o}+\delta_{1}, r_{o}$ is the initial closure at $\delta_{1}=0$. The differential form of the stress-deformation relationship given in equation (13) may be used to obtain creep and stiffness functions or complex compliances and stiffnesses. The complex form of stress-deformation relationship is given as:

$$
F_{i}(\omega)=R_{i j}(\omega) \Delta_{j}(\omega)
$$

The kinematic approach, typically, yields the following form of complex stiffness tensor:

$$
R_{i j}=C_{i j}+I \omega \eta_{i j}
$$

which is analogous to the Kelvin solid in visco-elasticity.

In the static approach, the overall interface displacement, $\Delta_{i}$, is obtained from the summation of the displacements, $\delta_{i}{ }^{c}$, developed at asperities, which for a large number of asperity contacts is written as the following integral:

$$
\Delta_{i}=N \iint_{r} \delta_{i}^{c} \xi(\Omega) H(r) d \Omega d r
$$

Asperity displacement, $\delta_{i}^{c}$, is obtained in terms of the asperity force, $f_{i}^{c}$, from the solution of the differential form of the asperity force-displacement relationship given in equation (5), as follows:

$$
\delta_{i}^{c}=\int_{0}^{t}\left(\frac{1-\exp \left(1-\frac{t}{t_{n}^{c}}\right)}{K_{n}^{c}} n_{i}^{c} n_{j}^{c}+\frac{1-\exp \left(1-\frac{t}{t_{s}^{c}}\right)}{K_{s}^{c}}\left(s_{i}^{c} s_{j}^{c}+t_{i}^{c} t_{j}^{c}\right)\right) \frac{d}{d t} f_{j}^{c} d t
$$

Under the static assumption the asperity force, $f_{i}^{c}$, is related to the overall traction of the interface, $F_{i}$, as follows:

$$
f_{i}^{c}=\frac{1}{N} F_{i}
$$

Thus, the overall interface displacement, $\Delta_{i}$, may be written as:

$$
\Delta_{i}=\frac{1}{N} \int_{0}^{t} J_{i j} \frac{d}{d t} F_{j} d t
$$

where interface creep function, denoted by, $J_{i j}$, is obtained as the following integral:

$$
J_{i j}=\iint_{r \Omega}\left(\frac{1-\exp \left(1-\frac{t}{t_{n}^{c}}\right)}{K_{n}^{c}} n_{i}^{c} n_{j}^{c}+\frac{1-\exp \left(1-\frac{t}{t_{s}^{c}}\right)}{K_{S}^{c}}\left(s_{i}^{c} s_{j}^{c}+t_{i}^{c} t_{j}^{c}\right) \xi(\Omega) H(r) d \Omega d r\right.
$$


The creep function given in equation (21) may be used to obtain stiffness functions or complex compliances and stiffnesses. The static approach, typically, yields the following form of complex stiffness tensor:

$$
R_{i j}=R_{i j}(\omega)+I \bar{R}_{i j}(\omega)
$$

\section{RESULTS FOR REFLECTION AND TRANSMISSION AT INTERFACES}

To illustrate the capabilities of the micromechanical model in elucidating the wave propagation phenomena we present two examples. We first use rate-independent effective stiffness derived from kinematic approach since: (1) many interfaces show weak rate dependence and (2) kinematic approach gives a reasonable prediction of the effective stiffness under quasi-static conditions. We then use rate dependent effective stiffness derived from static approach, since rate dependent behavior shown by the kinematic approach is a special case of the static approach.

We investigate the reflection and transmission of normally incident shear wave (propagating along 1-axis) to illustrate the effects of inherent anisotropy. In Fig. 3, we plot, in polar coordinates, the reflection and transmission coefficients asperity contact orientation parameter, $\mathrm{c}=0,0.1$ and 0.3 . The polar plots correspond to the incident shearwave's polarization direction given by the azimuthal angle, $\phi$. For instance, $\phi=0$ corresponds to the polarization direction along the 3 -axis. For $\mathrm{c}=0$, the normally incident shear wave does not suffer any mode conversion and the reflected and transmitted waves are independent of the incident wave polarization. For non-zero c-values, representing an anisotropic distribution of asperity contact orientation, the normally incident shear wave suffers mode conversion to transmitted and reflected SH waves. We also observe that the reflected and the transmitted of the mode converted waves are equal in amplitude. It is noteworthy that the amplitudes of the reflected and transmitted waves are dependent upon the direction of polarization of the incident waves. In this example, mode conversion to Pwave does not occur as the shear and normal stiffnesses are decoupled. However, under a small shear load on the interface, these stiffnesses would be coupled and a mode converted P-wave will be observed [9].

Under normal incidence of a P-wave, the amplitudes of the reflected and transmitted wave are given as

$$
\left|T_{p}\right|=\sqrt{\frac{4\left(R_{11}^{2}(\omega)+\bar{R}_{11}^{2}(\omega)\right)}{\left(4 R_{11}^{2}(\omega)+\omega^{2} Z_{p}^{2}-4 \omega Z_{p} \bar{R}_{11}(\omega)+4 \bar{R}_{11}^{2}(\omega)\right)}} \text { and }\left|R_{p}\right|=\sqrt{\frac{\omega^{2} Z_{p}^{2}}{\left(4 R_{11}^{2}(\omega)+\omega^{2} Z_{p}^{2}-4 \omega Z_{p} \bar{R}_{11}(\omega)+4 \bar{R}_{11}^{2}(\omega)\right)}}
$$
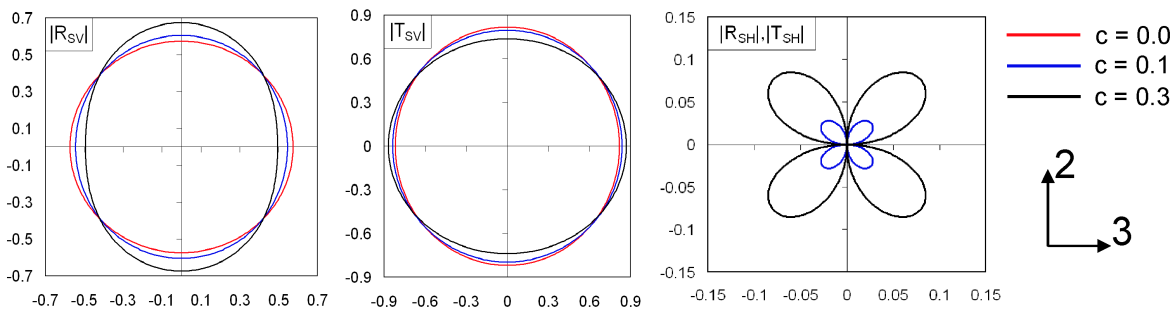

FIGURE 3. Mode conversion of SV to SH waves under normal incidence and normal closure. 

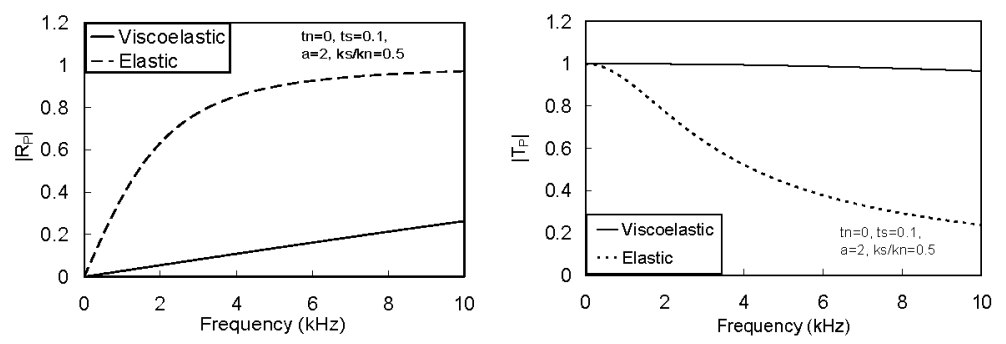

FIGURE 4. Effect of frequency upon reflected and transmitted wave amplitudes.

In Fig. 4 we have plotted the transmitted and reflected wave amplitudes computed using equation (23) for specific values of asperity stiffness constants, asperity orientation parameter, $a=2$, such that the mean asperity contact orientation is $29^{\circ}$, and asperity viscosity constants such that $t_{n}=\eta_{n} / K_{n}=0$ and $t_{s}=\eta_{s} / K_{s}=0.1$. For comparison, we have also plotted in Fig. 4, the transmitted and reflected wave amplitudes for a rate-independent interface with the same asperity stiffness constants and asperity orientation parameter. Significantly different behavior is exhibited by the rate-dependent interface over the same frequency range. Clearly, for the same roughness, the attenuation with frequency is much higher for rate-independent interface than rate-dependent interfaces.

\section{SUMMARY AND CONCLUSIONS}

The main findings of this work are summarized as follows:

1. Effective stiffness of interfaces between two rough solids are known to be affected by surface roughness, existing stress conditions and asperity contact elasticity and viscosity.

2. A micromechanical methodology for modeling rate-dependent stress-displacement behavior of such interfaces is presented. Complex moduli are derived using kinematic and static approaches. The model is then used to investigate plane wave propagation behavior through interfaces utilizing the well established imperfectly bonded interface model.

3. We find that the amplitudes of the reflected and transmitted waves are significantly influenced by the roughness and rate-dependence. The model may thus be used to elucidate the results of wave transmission through rough interfaces

\section{REFERENCES}

1. Kendal, K. and Tabor, D. Proc. R. Soc. London, A323, pp. 321-340, (1971).

2. Rokhlin, S.I. and Wang, Y.J. J. Acoust. Soc Am., 89, pp. 503-515, (1991).

3. Schoenberg, M. J. Acoust. Soc Am., 68, pp. 1516-1521, (1980).

4. Misra, A. J. Eng. Mech., 123, pp. 475-484, (1997).

5. Misra, A. J Geophys Res, 104, pp. 23,175-23,187, (1999).

6. Yoshioka, N. Tectonophysics, 277, pp. 29-40, (1997).

7. Adler, RJ, Firman, D. Phil Trans Royal Soc London, A303, pp.433-462, (1981).

8. Nayak, P.R., J. Lubr. Technol., 93, pp. 398-407, (1971).

9. Misra, A. and Marangos, O., "Application of a Micromechanical Model to Wave Propagation through Nonlinear Rough Interfaces Under Stress," in Proceedings 2006 IEEE International Ultrasonics Symposium, Vancouver, British Columbia, Canada, 2007, pp. 309-312. 